\title{
Triglyceride Glucose-Waist Circumference (TyG-WC) is a Reliable Marker to Predict Non-Alcoholic Fatty Liver Disease
}

\author{
Seungah Song \\ Gangnam Severance Hospital \\ Da-Hye Son \\ Gangnam Severance Hospital \\ Su Jung Baik \\ Gangnam Severance Hospital \\ Wan Je Cho \\ Gangnam Severance Hospital \\ Yong-Jae Lee ( $\sim$ ukyjhome@yuhs.ac ) \\ Gangnam Severance Hospital
}

\section{Research Article}

Keywords: NAFLD, TyG index, TyG-WC, TyG-BMI, fatty liver index, insulin resistance, prediction, diagnosis, body composition, Korean

Posted Date: January 17th, 2022

DOI: https://doi.org/10.21203/rs.3.rs-1216056/v1

License: (c) (i) This work is licensed under a Creative Commons Attribution 4.0 International License. Read Full License 


\section{Abstract}

Triglyceride and glucose index (TyG index), a marker of insulin resistance, is positively associated with NAFLD. Modified TyG indices, combining body composition markers including body-mass index (BMI) or waist circumference (WC) with TyG index, are reported to enhance predictability of insulin resistance. This study aimed to compare the usefulness of modified TyG indices for predicting NAFLD to TyG index and fatty liver index (FLI). This cross-sectional study included 12,757 Korean adults. TyG index and FLI were calculated using established formulas, and TyG-BMI and TyG-WC were calculated as TyG * BMI and TyG * WC, respectively. All measures were divided into quartiles. NAFLD severity (grade 0-3) was compared using ANOVA by quartiles of each index. Odds ratios (ORs) and 95\% confidence intervals (Cls) for NAFLD were calculated using multiple logistic regression analysis. ROC and AUROC analyses were performed to compare predictability of NAFLD using WC, BMI, TyG, TyG-BMI, TyG-WC and FLI. Higher TyG index, TyGBMI, TyG-WC, and FLI were associated with higher grade of NAFLD. ORs(Cls) for NAFLD increased in all indices, especially in TyG-WC [39.251 (31.304-49.215)] and FLI [38.937 (31.145-48.678)]. AUROC was $0.848(0.840-0.855)$ for TyG-WC and 0.850 (0.842-0.857) for FLI. TyG-WC is a reliable indicator for the presence of NAFLD in Korean adults.

\section{Introduction}

Non-alcoholic fatty liver disease (NAFLD) is characterized by the diffuse accumulation of triglycerides in hepatocytes not caused by excessive alcohol intake and other causes of liver disease. NAFLD is generally attributed to obesity-induced insulin resistance [1]. With the growing epidemic of obesity, NAFLD is one of the most prevalent causes of chronic liver disease, ranging from 25-45\% [2]. In Korea, the prevalence of NAFLD was between 10 and $15 \%$ in non-obese and between 55 and $70 \%$ in obese individuals [3, 4]. NAFLD encompasses a spectrum of clinical syndromes ranging from simple steatosis to non-alcoholic steatohepatitis that may progress to advanced fibrosis, cirrhosis, and cirrhosis complicated by hepatocellular carcinoma [5-8]. NAFLD is clinically important because of its association with an increased risk of type 2 diabetes, cardiovascular disease, and chronic kidney disease as well as liver-related diseases [7, 9-11]. Thus, early identification of individuals at higher risk for NAFLD may allow for the application of preventive strategies that can slow the morbidity and mortality of both liver-related and cardiometabolic diseases.

The traditional diagnosis of NAFLD requires various techniques such as liver ultrasonography, magnetic resonance, and biopsy [4]; however, these procedures are either invasive or expensive with limited applicability in general clinical practice. The general hypothesis is that, in NAFLD, an excess of visceral adipose tissue affects glucose metabolism and insulin resistance. Because of this, the fatty liver index (FLI), calculated using body mass index (BMI), waist circumference (WC), triglycerides (TG), and $\mathrm{Y}^{-}$ glutamyltransferase (GGT), has emerged as a simple and economical alternative tool for mass screening for hepatic steatosis with reasonable sensitivity and specificity [12]. In addition, a laboratory marker of insulin resistance, such as the triglyceride and glucose index (TyG index), has been positively associated with the presence of NAFLD [7, 13, 14]. More recently, several researchers have shown that the modified TyG index, which combines the TyG index with the effect of body composition including BMI or WC, enhances predictability of insulin resistance $[15,16]$. However, no large-scale studies have investigated the relationship between the modified TyG index and the presence of NAFLD. Therefore, this study aimed to compare the usefulness of the modified TyG index to that of the TyG index and FLI in predicting NAFLD.

\section{Results}

A total of 12,757 participants were enrolled in this study. Table 1 presents the clinical characteristics of the study population according to NAFLD status. There were 4,393 participants with NAFLD, among whom 2,769 (63.6\%) were male. The mean age in the group with NAFLD was $47.0 \pm 10.6$ years, and the group without NAFLD had a mean age of $44.8 \pm 11.6$ years. The mean age, BMl, WC, AST, ALT, fasting glucose, GGT, total cholesterol, HDL-C, LDL-C, and triglycerides were significantly higher in the study population with NAFLD than in the group with normal liver sonography. The proportion of smokers was also higher in the study population with NAFLD. 
Table 1

Characteristics of the study population

\begin{tabular}{|c|c|c|c|}
\hline & NAFLD (+) & NAFLD (-) & $p$-value \\
\hline $\mathrm{N}$ & 4393 & 8429 & \\
\hline Male & $2769(63.6)$ & $2532(30.1 \%)$ & $<0.001$ \\
\hline Age (years) & $47.00 \pm 10.61$ & $44.82 \pm 11.58$ & $<0.001$ \\
\hline $\mathrm{BMI}(\mathrm{kg} / \mathrm{m} 2)$ & $25.31 \pm 3.228$ & $21.88 \pm 2.637$ & $<0.001$ \\
\hline Waist circumference $(\mathrm{cm})$ & $85.53 \pm 8.873$ & $74.47 \pm 8.126$ & 0.001 \\
\hline Fasting glucose (mg/dL) & $100.61 \pm 17.99$ & $92.76 \pm 9.886$ & $<0.001$ \\
\hline AST (IU/L) & $30.10 \pm 19.41$ & $25.87 \pm 16.19$ & $<0.001$ \\
\hline ALT (IU/L) & $34.02 \pm 26.03$ & $20.08 \pm 14.70$ & $<0.001$ \\
\hline Alkaline phosphatase (IU/L) & $75.81 \pm 20.67$ & $66.93 \pm 21.19$ & 0.270 \\
\hline GGT (IU/L) & $33.68 \pm 32.07$ & $19.36 \pm 21.22$ & $<0.001$ \\
\hline Total cholesterol (mg/dL) & $216.98 \pm 36.97$ & $204.78 \pm 34.51$ & $<0.001$ \\
\hline HDL-cholesterol (mg/dL) & $52.43 \pm 11.59$ & $61.98 \pm 13.18$ & $<0.001$ \\
\hline LDL-cholesterol (mg/dL) & $141.55 \pm 29.70$ & $126.33 \pm 27.62$ & $<0.001$ \\
\hline Triglycerides $(\mathrm{mg} / \mathrm{dL})$ & $153.72 \pm 75.60$ & $97.60 \pm 47.92$ & $<0.001$ \\
\hline Current smoker (\%) & $823(20.1 \%)$ & $731(9.2 \%)$ & $<0.001$ \\
\hline Current exercise (\%) & $1395(33.5 \%)$ & $2646(30.3 \%)$ & $<0.001$ \\
\hline
\end{tabular}

Figure 2 showed the fatty liver severity according to quartiles of TyG index, TyG-BMI, TyG-WC, and FLI. The higher the indices, the higher was the fatty liver grade for all indices. The increase in the fatty liver severity was more pronounced in the TyG-WC index and FLI measurements.

The ORs ( $95 \% \mathrm{Cls}$ ) for NAFLD according to the quartiles of each parameter are shown in Table 2. Compared to the lowest TyG quartile in all participants, the OR $(95 \% \mathrm{Cl})$ for NAFLD was 9.036 (7.763-10.517) in the highest quartile after adjusting for age, AST, ALT, SBP, DBP, smoking, and exercise. The TyG-BMI index OR (95\% Cl) for NAFLD was 26.275 (21.710-31.799), the TyG-WC index OR (Cl) was 39.251 (31.304-49.215) for TyG-WC index, and the FLI OR (CI) was 38.937 (31.145-48.678). When stratified by gender, the ORs (95\% Cls) for NAFLD in male participants were 5.691 (4.686-6.913) for TyG index, 12.870 (10.339-16.021) for TyG-BMI, 14.050 (11.014-17.923) for TyG-WC, and 15.692 (12.078-20.387) for FLI. Similar results were observed in female participants: 8.659 (6.87410.908) for TyG index, 21.353 (16.1340-28.259) for TyG-BMI, 26.379 (19.220-36.205) for TyG-WC, and 22.518 (16.979-29.864) for FLI. 
Table 2

Odds ratios for NAFLD according to quartiles of each parameter.

\begin{tabular}{|c|c|c|c|c|c|c|c|c|c|c|c|c|}
\hline & \multicolumn{4}{|c|}{ All subjects } & \multicolumn{4}{|c|}{ Male } & \multicolumn{4}{|c|}{ Female } \\
\hline & Q1 & Q2 & Q3 & Q4 & Q1 & Q2 & Q3 & Q4 & Q1 & Q2 & Q3 & Q4 \\
\hline \multicolumn{13}{|l|}{ TyG index } \\
\hline \multirow[t]{2}{*}{ Unadjusted } & 1 & 2.422 & 5.313 & 18.150 & 1 & 2.098 & 4.280 & 9.322 & 1 & 2.188 & 4.385 & 13.646 \\
\hline & & $\begin{array}{l}(2.102- \\
2.791)\end{array}$ & $\begin{array}{l}(4.643- \\
6.079)\end{array}$ & $\begin{array}{l}(15.841- \\
20.794)\end{array}$ & & $\begin{array}{l}(1.784- \\
2.467)\end{array}$ & $\begin{array}{l}(3.634- \\
5.039)\end{array}$ & $\begin{array}{l}(7.815- \\
11.120)\end{array}$ & & $\begin{array}{l}(1.723- \\
2.779)\end{array}$ & $\begin{array}{l}(3.509- \\
5.479)\end{array}$ & $\begin{array}{l}(11.020- \\
16.899)\end{array}$ \\
\hline \multirow[t]{2}{*}{ Adjusted } & 1 & 2.048 & 3.652 & 9.036 & 1 & 1.812 & 3.315 & 5.691 & 1 & 1.881 & 3.507 & 8.659 \\
\hline & & $\begin{array}{l}(1.759- \\
2.384)\end{array}$ & $\begin{array}{l}(3.153- \\
4.230)\end{array}$ & $\begin{array}{l}(7.763- \\
10.517)\end{array}$ & & $\begin{array}{l}(1.523- \\
2.156)\end{array}$ & $\begin{array}{l}(2.777- \\
3.957)\end{array}$ & $\begin{array}{l}(4.686- \\
6.913)\end{array}$ & & $\begin{array}{l}(1.461- \\
2.421)\end{array}$ & $\begin{array}{l}(2.768- \\
4.443)\end{array}$ & $\begin{array}{l}\text { (6.874- } \\
10.908)\end{array}$ \\
\hline \multicolumn{13}{|l|}{ TyG-BMI } \\
\hline \multirow[t]{2}{*}{ Unadjusted } & 1 & 3.493 & 12.450 & 49.701 & 1 & 2.889 & 6.943 & 22.089 & 1 & 2.228 & 6.559 & 30.635 \\
\hline & & $\begin{array}{l}(2.919- \\
4.181)\end{array}$ & $\begin{array}{l}(10.515- \\
14.742)\end{array}$ & $\begin{array}{l}(41.819- \\
59.069)\end{array}$ & & $\begin{array}{l}(2.431- \\
3.434)\end{array}$ & $\begin{array}{l}(5.833- \\
8.265)\end{array}$ & $\begin{array}{l}(18.080- \\
26.987)\end{array}$ & & $\begin{array}{l}(1.658- \\
2.994)\end{array}$ & $\begin{array}{l}(5.019- \\
8.570)\end{array}$ & $\begin{array}{l}(23.652- \\
39.680)\end{array}$ \\
\hline \multirow[t]{2}{*}{ Adjusted } & 1 & 3.057 & 9.067 & 26.275 & 1 & 2.477 & 5.248 & 12.870 & 1 & 2.041 & 5.410 & 21.353 \\
\hline & & $\begin{array}{l}(2.528- \\
3.697)\end{array}$ & $\begin{array}{l}(7.556- \\
10.880)\end{array}$ & $\begin{array}{l}(21.710- \\
31.799)\end{array}$ & & $\begin{array}{l}(2.069- \\
2.965)\end{array}$ & $\begin{array}{l}(4.364- \\
6.310)\end{array}$ & $\begin{array}{l}(10.339- \\
16.021)\end{array}$ & & $\begin{array}{l}(1.493- \\
2.789)\end{array}$ & $\begin{array}{l}(4.066- \\
7.198)\end{array}$ & $\begin{array}{l}(16.134- \\
28.259)\end{array}$ \\
\hline \multicolumn{13}{|l|}{ TyG-WC } \\
\hline \multirow[t]{2}{*}{ Unadjusted } & 1 & 3.733 & 14.018 & 60.054 & 1 & 2.745 & 8.022 & 23.563 & 1 & 2.360 & 6.295 & 34.604 \\
\hline & & $\begin{array}{l}(3.047- \\
4.574)\end{array}$ & $\begin{array}{l}\text { (11.580- } \\
16.971)\end{array}$ & $\begin{array}{l}\text { (49.396- } \\
73.011)\end{array}$ & & $\begin{array}{l}(2.274- \\
3.313)\end{array}$ & $\begin{array}{l}(6.634- \\
9.701)\end{array}$ & $\begin{array}{l}(18.975- \\
29.261)\end{array}$ & & $\begin{array}{l}(1.704- \\
3.267)\end{array}$ & $\begin{array}{l}(4.676- \\
8.474)\end{array}$ & $\begin{array}{l}(25.987- \\
46.079)\end{array}$ \\
\hline \multirow[t]{2}{*}{ Adjusted } & 1 & 3.638 & 11.936 & 39.251 & 1 & 2.466 & 6.311 & 14.050 & 1 & 2.243 & 5.602 & 26.379 \\
\hline & & $\begin{array}{l}(2.930- \\
4.518)\end{array}$ & $\begin{array}{l}\text { (9.673- } \\
14.727)\end{array}$ & $\begin{array}{l}(31.304- \\
49.215)\end{array}$ & & $\begin{array}{l}(2.020- \\
3.011)\end{array}$ & $\begin{array}{l}(5.143- \\
7.793)\end{array}$ & $\begin{array}{l}\text { (11.014- } \\
17.923)\end{array}$ & & $\begin{array}{l}(1.589- \\
3.166)\end{array}$ & $\begin{array}{l}(4.070- \\
7.711)\end{array}$ & $\begin{array}{l}(19.220 \\
-36.205)\end{array}$ \\
\hline \multicolumn{13}{|l|}{ FLI } \\
\hline \multirow[t]{2}{*}{ Unadjusted } & 1 & 3.655 & 14.263 & 58.953 & 1 & 3.108 & 8.371 & 27.765 & 1 & 1.658 & 5.152 & 28.139 \\
\hline & & $\begin{array}{l}(2.986- \\
4.474)\end{array}$ & $\begin{array}{l}(11.796- \\
17.248)\end{array}$ & $\begin{array}{l}(48.542- \\
71.597)\end{array}$ & & $\begin{array}{l}(2.566- \\
3.766)\end{array}$ & $\begin{array}{l}(6.940- \\
10.098)\end{array}$ & $\begin{array}{l}(22.078- \\
34.918)\end{array}$ & & $\begin{array}{l}(1.211- \\
2.270)\end{array}$ & $\begin{array}{l}(3.910- \\
6.788)\end{array}$ & $\begin{array}{l}(21.622- \\
36.618)\end{array}$ \\
\hline \multirow[t]{2}{*}{ Adjusted } & 1 & 3.495 & 12.494 & 38.937 & 1 & 2.875 & 6.645 & 15.692 & 1 & 1.480 & 4.735 & 22.518 \\
\hline & & $\begin{array}{l}(2.823- \\
4.326)\end{array}$ & $\begin{array}{l}(10.172- \\
15.346)\end{array}$ & $\begin{array}{l}(31.145- \\
48.678)\end{array}$ & & $\begin{array}{l}(2.352- \\
3.515)\end{array}$ & $\begin{array}{l}(5.426- \\
8.139)\end{array}$ & $\begin{array}{l}(12.078- \\
20.387)\end{array}$ & & $\begin{array}{l}(1.065- \\
2.058)\end{array}$ & $\begin{array}{l}(3.549- \\
6.317)\end{array}$ & $\begin{array}{l}(16.979- \\
29.864)\end{array}$ \\
\hline
\end{tabular}

The predictability of NAFLD through WC, BMI, TyG, TyG-BMI, TyG-WC, and FLI are compared in Table 3 by areas under the ROC curves, illustrated in Figure3. The AUC was 0.805 (0.797-0.813) for BMI, 0.823 (0.815-0.831) for WC, 0.780 (0.771-0.789) for TyG index, 0.840 (0.832-0.847) for TyG-BMI, 0.848 (0.840-0.855) for TyG-WC, and 0.850 (0.842-0.857) for FLI. The AUC of TyG-WC and FLI were not statistically different with the post-hoc $p$-value of 0.1621 , and TyG-WC and FLI showed better predictability for NAFLD compared to other indices. 
Table 3

Areas under the ROC curves for each parameter for predicting NAFLD

\begin{tabular}{|c|c|c|c|c|c|c|c|}
\hline Parameters & AUC (95\% Cl) & Overall $p$-value & \multicolumn{5}{|c|}{ Post hoc $p$-value } \\
\hline \multicolumn{8}{|l|}{ All subjects } \\
\hline BMI & $0.805(0.797-0.813)$ & \multirow[t]{6}{*}{$<0.0001$} & \multicolumn{5}{|l|}{ Ref } \\
\hline WC & $0.823(0.815-0.831)$ & & $<0.0001$ & \multicolumn{4}{|l|}{ Ref } \\
\hline TyG index & $0.780(0.771-0.789)$ & & $<0.0001$ & $<0.0001$ & \multicolumn{3}{|l|}{ Ref } \\
\hline TyG-BMI & $0.840(0.832-0.847)$ & & $<0.0001$ & $<0.0001$ & $<0.0001$ & \multicolumn{2}{|l|}{ Ref } \\
\hline TyG-WC & $0.848(0.840-0.855)$ & & $<0.0001$ & $<0.0001$ & $<0.0001$ & $<0.0001$ & Ref \\
\hline FLI & $0.850(0.842-0.857)$ & & $<0.0001$ & $<0.0001$ & $<0.0001$ & $<0.0001$ & 0.1621 \\
\hline \multicolumn{8}{|l|}{ Male } \\
\hline BMI & $0.744(0.731-0.758)$ & & Ref & & & & \\
\hline WC & $0.762(0.749-0.776)$ & & $<0.0001$ & Ref & & & \\
\hline TyG index & $0.728(0.714-0.743)$ & \multirow[t]{4}{*}{$<0.0001$} & $<0.0001$ & $<0.0001$ & Ref & & \\
\hline TyG-BMI & $0.788(0.775-0.800)$ & & $<0.0001$ & $<0.0001$ & $<0.0001$ & Ref & \\
\hline TyG-WC & $0.798(0.786-0.811)$ & & $<0.0001$ & $<0.0001$ & $<0.0001$ & 0.0014 & Ref \\
\hline FLI & $0.800(0.787-0.812)$ & & $<0.0001$ & $<0.0001$ & $<0.0001$ & 0.0001 & 0.618 \\
\hline \multicolumn{8}{|l|}{ Female } \\
\hline BMI & $0.791(0.777-0.804)$ & & Ref & & & & \\
\hline WC & $0.804(0.791-0.817)$ & & 0.0008 & Ref & & & \\
\hline TyG index & $0.766(0.752-0.781)$ & \multirow[t]{4}{*}{$<0.0001$} & 0.0040 & $<0.0001$ & Ref & & \\
\hline TyG-BMI & $0.827(0.814-0.839)$ & & $<0.0001$ & $<0.0001$ & $<0.0001$ & Ref & \\
\hline TyG-WC & $0.836(0.823-0.848)$ & & $<0.0001$ & $<0.0001$ & $<0.0001$ & 0.0036 & Ref \\
\hline FLI & $0.834(0.822-0.847)$ & & $<0.0001$ & $<0.0001$ & $<0.0001$ & 0.0053 & 0.5569 \\
\hline
\end{tabular}

\section{Discussion}

In this cross-sectional study, different parameters were tested to predict the presence of NAFLD. A greater value of TyG-WC and FLI was associated with a higher grade of NAFLD. When divided into quartiles, the highest quartiles of TyG-WC and FLI showed greater ORs for NAFLD compared to the lowest quartiles than other parameters. Additionally, the AUROCs of TyG-WC and FLI were greater than the other parameters with no significant difference when compared to each other.

The gold standard for diagnosis for NAFLD is liver biopsy. However, since performing such an invasive procedure on a large number of people is unreasonable, serum biomarkers and abdominal sonographies are usually used for diagnosis. Many attempts were targeted at finding an appropriate marker for NAFLD, one of which was FLI [12]. FLI involves measurements included in routine lab chemistries as well as easily obtainable BMI and WC. In addition, FLI was associated with a high AUROC of 0.86 in a Korean population study [3]; this result suggests that FLI is a promising diagnostic tool for NAFLD. However, the complicated calculation formula hinders its widespread use in clinical practice. Recent studies have shown that TyG index, a marker initially developed as an alternative index for insulin resistance, could be used as a diagnostic tool for NAFLD instead of FLI [7]. TyG index has a simpler formula than FLI and has been widely used in many recent studies as well as in clinical practice [20-22]. 
However, Khamseh et al. suggested that the TyG index with the addition of indices of obesity such as BMI and WC may be more accurate than TyG index alone [14]. TyG-WC and TyG-BMI are also simply calculated by multiplying TyG index by WC and BMI, respectively. The goal of their study was to evaluate TyG-index, TyG-WC, and TyG-BMI for their prediction of NAFLD and liver fibrosis in overweight/obese individuals. The AUROCs in their study was 0.676 for TyG index, 0.675 for TyG-BMI, and 0.693 for TyG-WC, all of which are lower than the AUROCs produced in our study. The difference may be due to the fact that our study was conducted with a large study sample; their study had only 184 overweight/obese participants. Additionally, we were able to compare the values of the different parameters with the participants' abdominal sonography readings. We were able to find that higher values of all indices related to higher grades of fatty liver (Figure 2).

There are several possible explanations for our findings that TyG-BMI and TyG-WC were better predictors than TyG index alone. Accounting for the body composition may be essential in diseases related to metabolic syndrome, including NAFLD, which is metabolic syndrome's hepatic manifestation. More specifically, our study showed that TyG-WC was superior to TyG-BMI in predicting NAFLD. WC is relatively more representative of visceral fat deposition, and BMI is considered a more general obesity marker that takes subcutaneous fat deposition into account [23]. The distribution of fat is more important than the total fat mass. Increased visceral fat in the abdomen is more related to insulin resistance and metabolic dysfunction, including hepatic steatosis, than subcutaneous fat [24-26]. This may explain our study results.

This study has several limitations. First, this was a single center study with a single ethnic cohort of Korean adults. Second, there is potential for selection bias as study participants were self-referred for health screenings. Third, this study had a retrospective crosssectional design. Future studies should include heterogeneous populations to ensure generalizability to other ethnicities. In addition, multi-centered longitudinal cohort studies should be conducted to determine whether high values in these parameters predict future occurrences of NAFLD. Despite these potential limitations, our study is unique in that TyG-WC showed similar predictability for NAFLD to FLI, a specific diagnostic marker for NAFLD. To the best of our knowledge, this is the first study to compare predictability for NAFLD between modified TyG indices and FLI.

In conclusion, this study showed that TyG-WC can be a useful screening tool for NAFLD in Korean adults. TyG-WC has the advantages of being easily obtained and calculated with higher predictability compared to FLI.

\section{Materials And Methods Study participants}

This study was conducted with data from regular health examinations at the Health Promotion Center, Gangnam Severance Hospital, Yonsei University College of Medicine between January 2017 and October 2020. Of the 27,554 participants enrolled in the survey during the study period, 24,154 Koreans of age 20 years and older took part. From these participants, we excluded those meeting at least one of following criteria $(n=11,397)$ : those without abdominal sonography results $(n=149)$; those with a known history of cancer or viral hepatitis $(n=1,503)$; those taking medication for hypertension, diabetes mellitus, and dyslipidemia $(n=4,452)$; those with liver cirrhosis observed via ultrasound $(n=25)$; those with triglycerides $>500 \mathrm{mg} / \mathrm{dL}(n=78)$, and heavy consumers of alcohol ( $n$ $=5,190$ ). After these exclusions, the final analysis comprised data from 12,757 participants (Figure 1). Each patient who was a part of the trial gave their written informed consent. This study was conducted in accordance with the ethical principles of the Declaration of Helsinki and was approved by the Institutional Review Board of Yonsei University College of Medicine, Seoul, Korea (IRB number: 32021-0093)

\section{Data collection}

Each participant filled out and provided a self-administered survey that inquired on aspects on their social and medical history, such as smoking, alcohol intake, physical activity, medications, and previous disease history. Smoking status was categorized as nonsmoker, ex-smoker, or current smoker. Excessive alcohol drinking was defined as at least twice a week usage. Regular exercise was defined as exercise at least three times a week. Weight and height were measured to the nearest $0.1 \mathrm{~kg}$ and $0.1 \mathrm{~cm}$, respectively, in light-weighing indoor clothing with bare feet. The body mass index (BMI) was computed by dividing the weight in kilograms divided by the square of the height in meters $(\mathrm{kg} / \mathrm{m} 2)$. Systolic blood pressure (SBP) and diastolic blood pressure (DBP) were measured using the patient's right arm and a standard mercury sphygmomanometer (Baumanometer, W.A. Baum Co Inc., Copiague, NY, USA) 
after five minutes of rest. After a 12-hour overnight fast, all blood samples were obtained from the antecubital vein. Fasting plasma glucose, triglycerides, high-density lipoprotein cholesterol (HDL-C), low-density lipoprotein cholesterol (LDL-C), aspartate aminotransferase (AST), alanine aminotransferase (ALT), and GGT levels were measured by enzymatic methods using a chemistry analyzer (Hitachi 7600, Hitachi Co., Tokyo, Japan). SBP of $140 \mathrm{mmHg}$, DBP of $90 \mathrm{mmHg}$, or current usage of hypertension medication were all considered hypertension. A fasting plasma glucose level of $126 \mathrm{mg} / \mathrm{dL}$ or current usage of hypertensive medication were used to determine type 2 diabetes. Triglycerides of $150 \mathrm{mg} / \mathrm{dL}$ or less, low HDL-cholesterol of $50 \mathrm{mg} / \mathrm{dL}$, or current usage of dyslipidemia medicines were all considered dyslipidemia. Positive results for hepatitis $B$ surface antigen or anti-hepatitis $C$ virus antibody were used to define viral hepatitis. A Roche E-170 equipment was used to test the amounts of hepatitis B surface antigen and anti-hepatitis $\mathrm{C}$ viral antibodies (Roche Diagnostics, Mannheim, Germany).

\section{FLI, TyG index and TyG related parameters}

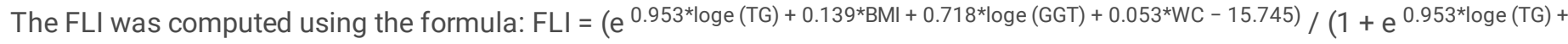
$0.139 * \mathrm{BMI}+0.718 * \operatorname{loge}(\mathrm{GGT})+0.053 * \mathrm{WC}-15.745) * 100[12]$.

The TyG index was calculated using the formula: Tyg Index = In [fasting TGs (mg/dL) * fasting glucose (mg/dL)/2] [17]. TyG-WC and TyG-BMI are defined as TyG index * WC and TyG index * BMI, respectively [18].

\section{NAFLD}

Fatty liver disease was diagnosed based on the findings of an abdominal ultrasonography scan with a 3.5-MHz transducer (HDI 5000 , Philips, Bothell, WA, USA). Ultrasonography was carried out by two experienced radiologists who were unaware of the aims of the study and blinded to laboratory findings; the coefficients of variations for inter- and intra-operator reproducibility were $6.8 \%$ and $4.3 \%$, respectively. In this investigation, livers with any degree of hepatic steatosis were considered to have NAFLD. The severity of NAFLD was classified into four grades: grade 0 (absence of steatosis with normal echogenicity), grade 1 (slight diffuse increase in bright homogeneous echoes in the liver parenchyma with normal visualization of the diaphragm and portal and hepatic vein borders and normal hepatorenal contrast), grade 2 (moderate steatosis, impaired echogenicity of the main portal vein wall), and grade 3 (marked increase in bright echoes at a shallow depth with deep attenuation and impaired visualization of the diaphragm and marked vascular blurring) [19].

\section{Statistical analysis}

Clinical characteristics of the study population were compared using an independent two sample t-test; chi-square tests were used for categorical variables. Fatty liver severity was compared using a one-way analysis of variance (ANOVA) test according to the quartile of each index. The odds ratios (ORs) and 95\% confidence intervals (Cls) for NAFLD were calculated using multiple logistic regression analysis after adjusting for confounding variables, including AST, ALT, SBP, DBP, smoking status, and exercise status, across TyG and TyG-related parameter quartiles. Receiver operating characteristic (ROC) and area under the curve (AUC) analyses were performed without adjustment to compare the predictability of NAFLD between WC, BMI, TyG, TyG-BMI, TyG-WC, and FLI. Comparison of ROCs was performed using the Delong method. All analyses were conducted using the SPSS software (version 25; IBM Corp., Armonk, NY, USA). All statistical tests were two-tailed, and statistical significance was determined at $p<0.05$.

\section{Declarations}

\section{Author contributions}

Conceptualization: S.-A.S and D.-H.S; methodology: D.-H.S; validation: D.-H.S; formal analysis: D.-H.S; investigation: SJ.B and WJ.C; resources: SJ.B and WJ.C; data curation: SJ.B and WJ.C; writing-original draft preparation: S.-A.S and D.-H.S; writing-review and editing: Y.-J.L ; visualization: S.-A.S. All authors have read and agreed to the published version of the manuscript.

\section{Competing interest}

The authors declare that they have no known competing interests.

\section{Funding}


The authors declare that no funds, grants, or other support were received during the preparation of this manuscript.

\section{Data availability}

The datasets generated during and/or analyzed during the current study are available from the corresponding author on reasonable request.

\section{Consent to publish}

Not applicable.

\section{Plant reproducibility}

Not applicable.

\section{Clinical trials registration}

Not applicable.

\section{References}

1. Lee YH, Bang H, Park YM, Bae JC, Lee BW, Kang ES, et al. Non-laboratory-based self-assessment screening score for nonalcoholic fatty liver disease: development, validation and comparison with other scores. PLoS One. 2014;9(9):e107584. https://doi.org/10.1371/journal.pone.0107584

2. Williams CD, Stengel J, Asike MI, Torres DM, Shaw J, Contreras M, et al. Prevalence of nonalcoholic fatty liver disease and nonalcoholic steatohepatitis among a largely middle-aged population utilizing ultrasound and liver biopsy: a prospective study. Gastroenterology. 2011;140(1):124-131.

3. Lee Y-h. Diagnosis of Non-Alcoholic Fatty Liver Disease Based on Clinical and Laboratory Data. J Korean Diabetes. 2017;18:7. https://doi.org/https://doi.org/10.4093/jkd.2017.18.2.102

4. Rinella ME. Nonalcoholic fatty liver disease: a systematic review. JAMA. 2015;313(22):2263-2273.

5. Kleiner DE, Brunt EM, Van Natta M, Behling C, Contos MJ, Cummings OW, et al. Design and validation of a histological scoring system for nonalcoholic fatty liver disease. Hepatology. 2005;41(6):1313-1321.

6. Singh S, Allen AM, Wang Z, Prokop LJ, Murad MH, Loomba R. Fibrosis progression in nonalcoholic fatty liver vs nonalcoholic steatohepatitis: a systematic review and meta-analysis of paired-biopsy studies. Clin. Gastroenterol. Hepatol. 2015;13(4):643654. e649.

7. Aya Kitae YH, Masahide Hamaguchi, Akihiro Obora, Takao Kojima, Michiaki Fukui. The Triglyceride and Glucose Index Is a Predictor of Incident Nonalcoholic Fatty Liver Disease: A Population-Based Cohort Study. Can. J. Gastroenterol. Hepatol. 2019;2019:7. https://doi.org/10.1155/2019/5121574

8. Pais R, Charlotte F, Fedchuk L, Bedossa P, Lebray P, Poynard T, et al. A systematic review of follow-up biopsies reveals disease progression in patients with non-alcoholic fatty liver. J. Hepatol. 2013;59(3):550-556.

9. Caussy C, Aubin A, Loomba R. The Relationship Between Type 2 Diabetes, NAFLD, and Cardiovascular Risk. Curr Diab Rep. 2021;21(5):1-13.

10. Byrne CD, Targher G. NAFLD as a driver of chronic kidney disease. J. Hepatol. 2020;72(4):785-801.

11. Bonora E, Targher G. Increased risk of cardiovascular disease and chronic kidney disease in NAFLD. Nat. Rev. Gastroenterol. Hepatol. 2012;9(7):372-381.

12. Bedogni G, Bellentani S, Miglioli L, Masutti F, Passalacqua M, Castiglione A, et al. The Fatty Liver Index: a simple and accurate predictor of hepatic steatosis in the general population. BMC Gastroenterol. 2006;6(1):1-7.

13. Zhang S, Du T, Zhang J, Lu H, Lin X, Xie J, et al. The triglyceride and glucose index (TyG) is an effective biomarker to identify nonalcoholic fatty liver disease. Lipids Health Dis. 2017;16(1):15. https://doi.org/10.1186/s12944-017-0409-6

14. Khamseh ME, Malek M, Abbasi R, Taheri H, Lahouti M, Alaei-Shahmiri F. Triglyceride Glucose Index and Related Parameters (Triglyceride Glucose-Body Mass Index and Triglyceride Glucose-Waist Circumference) Identify Nonalcoholic Fatty Liver and Liver 
Fibrosis in Individuals with Overweight/Obesity. Metab Syndr Relat Disord. 2021;19(3):167-173.

https://doi.org/10.1089/met.2020.0109

15. Khamseh ME, Malek M, Abbasi R, Taheri H, Lahouti M, Alaei-Shahmiri F. Triglyceride glucose index and related parameters (triglyceride glucose-body mass index and triglyceride glucose-waist circumference) identify nonalcoholic fatty liver and liver fibrosis in individuals with overweight/obesity. Metab Syndr Relat Disord. 2021;19(3):167-173.

16. Zheng S, Shi S, Ren X, Han T, Li Y, Chen Y, et al. Triglyceride glucose-waist circumference, a novel and effective predictor of diabetes in first-degree relatives of type 2 diabetes patients: cross-sectional and prospective cohort study. J. Transl. Med. 2016;14(1):1-10.

17. Guerrero-Romero F, Simental-Mendía LE, González-Ortiz M, Martínez-Abundis E, Ramos-Zavala MG, Hernández-González SO, et al. The product of triglycerides and glucose, a simple measure of insulin sensitivity. Comparison with the euglycemichyperinsulinemic clamp. J Clin Endocrinol Metab. 2010;95(7):3347-3351. https://doi.org/10.1210/jc.2010-0288

18. Zheng S, Shi S, Ren X, Han T, Li Y, Chen Y, et al. Triglyceride glucose-waist circumference, a novel and effective predictor of diabetes in first-degree relatives of type 2 diabetes patients: cross-sectional and prospective cohort study. J Transl Med. 2016;14:260. https://doi.org/10.1186/s12967-016-1020-8

19. Kang BK, Kim M, Shin SJ, Kim YJ. Correlation of Clinical and Histopathologic Parameters with Ultrasonographic Grades in Pediatric Non-Alcoholic Fatty Liver Disease. J Korean Med Sci. 2019;34(47):e298. https://doi.org/10.3346/jkms.2019.34.e298

20. Khan SH, Sobia F, Niazi NK, Manzoor SM, Fazal N, Ahmad F. Metabolic clustering of risk factors: evaluation of Triglycerideglucose index (TyG index) for evaluation of insulin resistance. Diabetol. metab. syndr. 2018;10(1):1-8.

21. Correa TL, Guelli MSTC, de Oliveira IO. Triglyceride-glucose Index (TyG) is Positively Associated with Nonalcoholic Fatty Liver Disease. Am. Heart J. 2021;242:158-159.

22. Brito ADMd, Hermsdorff HHM, Filgueiras MDS, Suhett LG, Vieira-Ribeiro SA, Franceschini SdCC, et al. Predictive capacity of triglyceride-glucose (TyG) index for insulin resistance and cardiometabolic risk in children and adolescents: a systematic review. Crit Rev Food. 2021;61(16):2783-2792.

23. Lim J, Kim J, Koo SH, Kwon GC. Comparison of triglyceride glucose index, and related parameters to predict insulin resistance in Korean adults: An analysis of the 2007-2010 Korean National Health and Nutrition Examination Survey. PLoS One. 2019;14(3):e0212963. https://doi.org/10.1371/journal.pone.0212963

24. Kissebah AH, Vydelingum N, Murray R, Evans DJ, Hartz AJ, Kalkhoff RK, et al. Relation of body fat distribution to metabolic complications of obesity. J Clin Endocrinol Metab. 1982;54(2):254-260. https://doi.org/10.1210/jcem-54-2-254

25. Arner P. Not all fat is alike. Lancet. 1998;351(9112):1301-1302.

26. Wajchenberg BL. Subcutaneous and visceral adipose tissue: their relation to the metabolic syndrome. Endocr Rev. 2000;21(6):697-738.

\section{Figures}




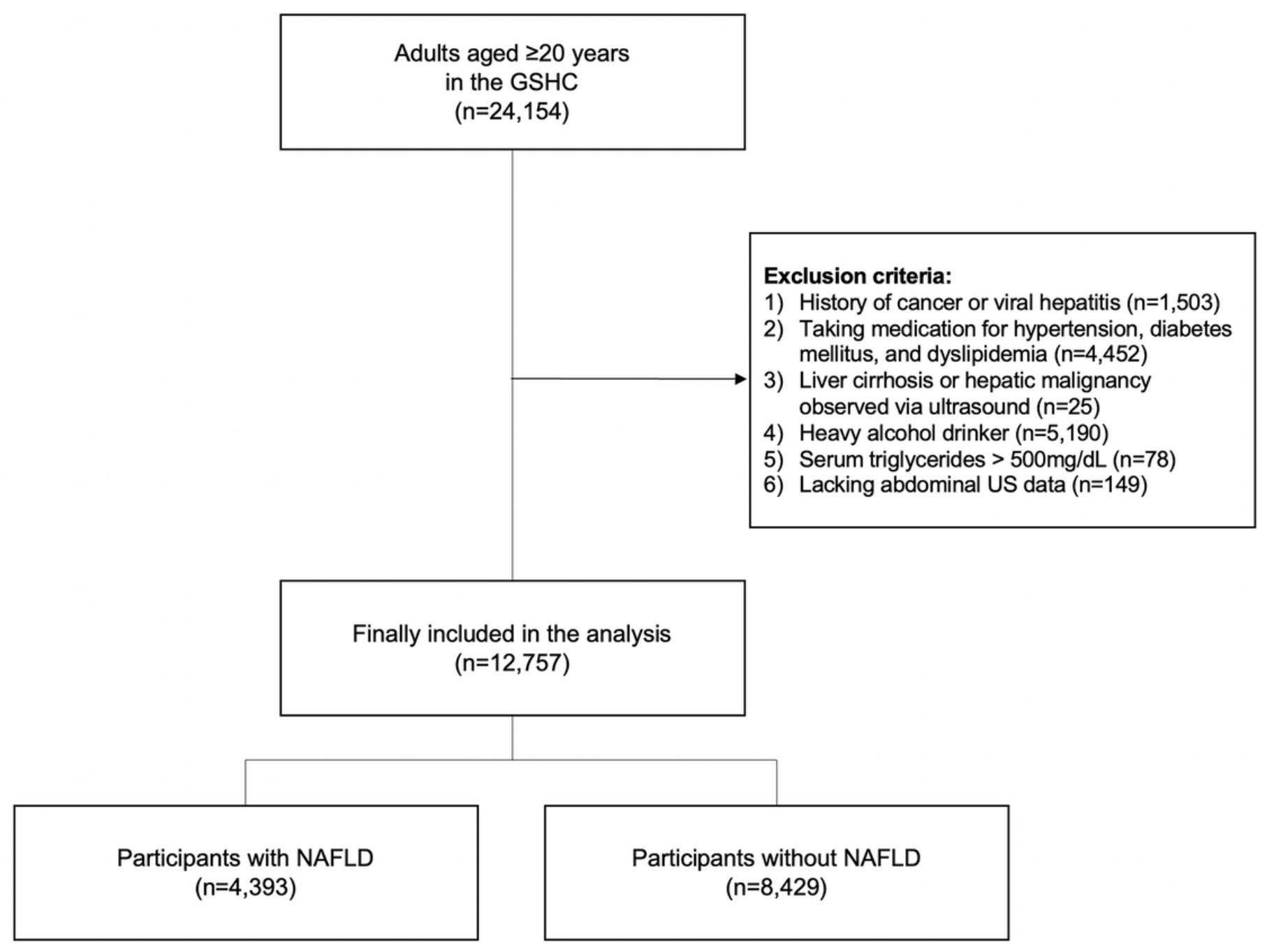

Figure 1

Study flow chart 
(A)

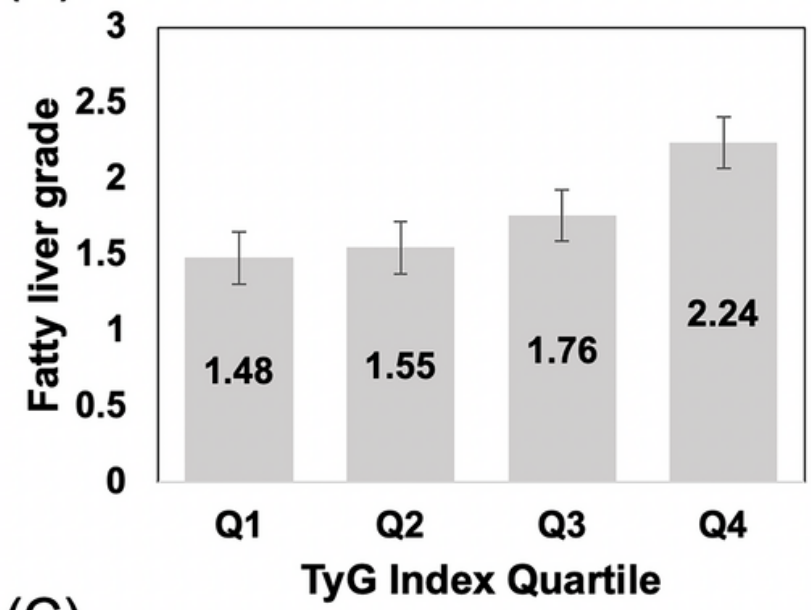

(C)

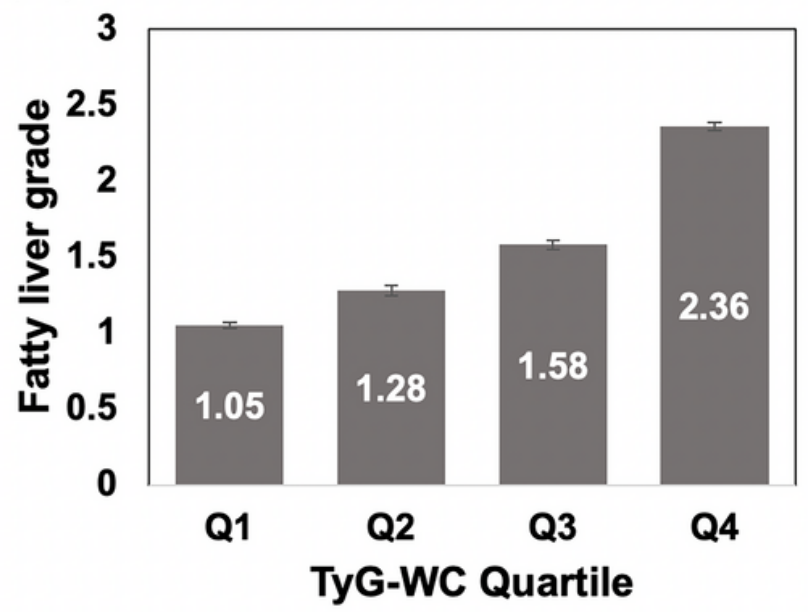

(B)

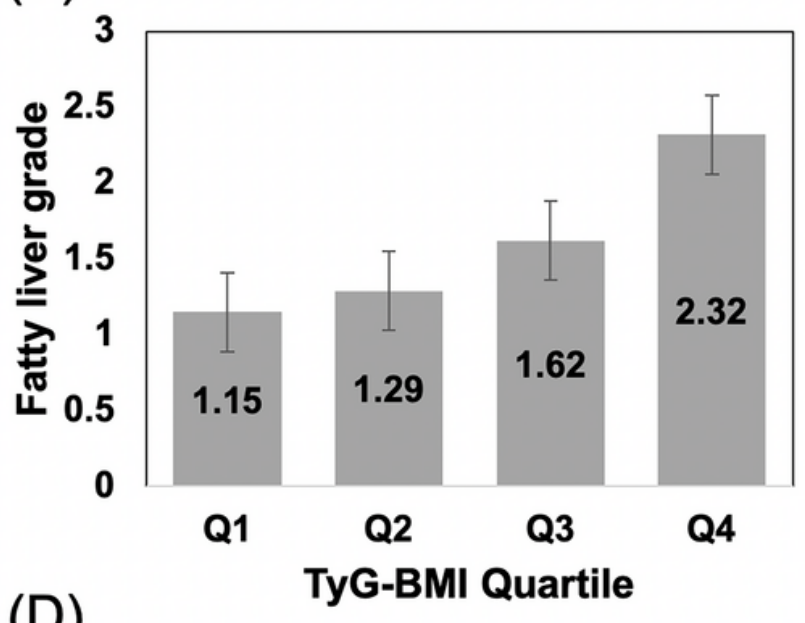

(D)

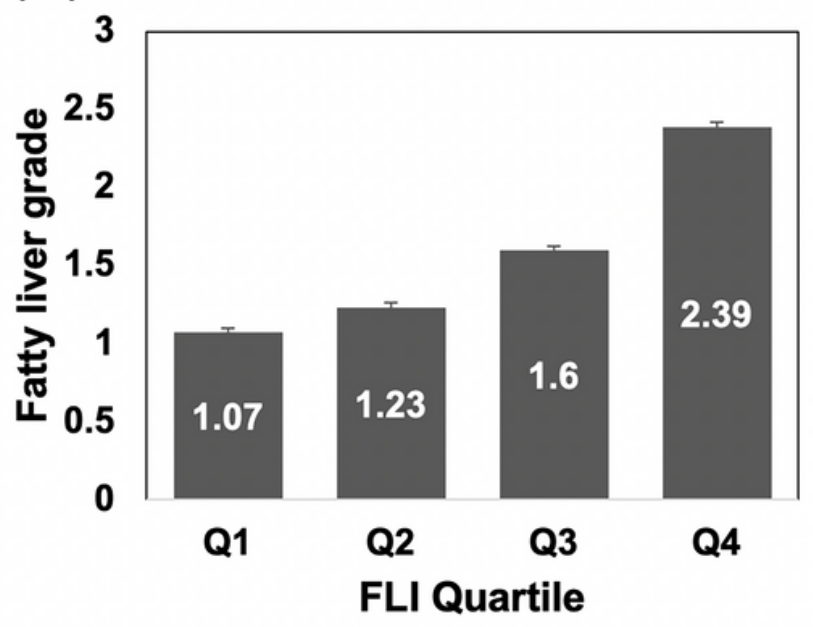

Figure 2

Fatty liver severity according to quartiles of each parameter. TyG, triglyceride and glucose; TyG-BMI, TyG-body mass index; TyG-WC, TyG-waist circumference; FLI, fatty liver index. 

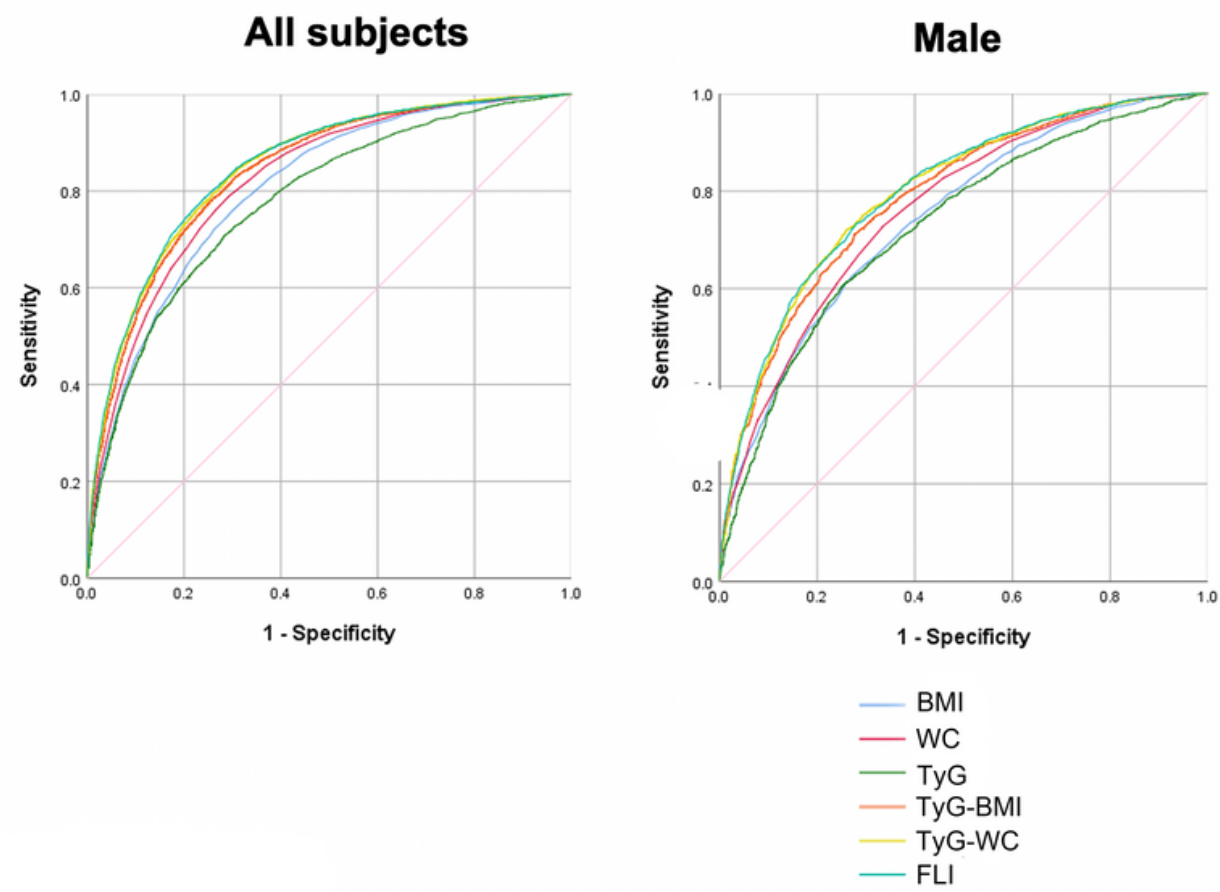

Female

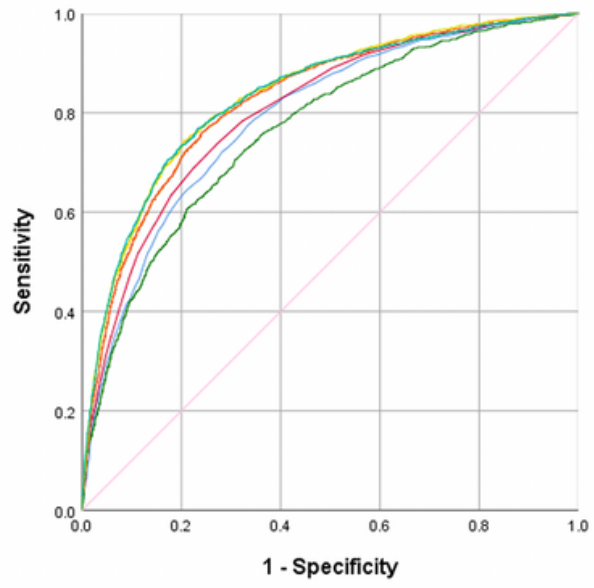

\section{Figure 3}

Receiver operating characteristic (ROC) curve of each parameter for predicting NAFLD. BMI, body mass index; WC, waist circumference; TyG, triglyceride and glucose; FLI, fatty liver index. 